\title{
Overview of chelation recommendations for thalassaemia and sickle cell disease
}

\author{
Banu Kaya \\ Barts Health NHS Trust, Royal London Hospital, London, UK
}

\begin{abstract}
The long term consequences of iron toxicity are mostly reversible with effective iron chelation therapy. Recommendations for use of chelation therapy in transfusion dependent thalassaemia (TDT), sickle cell disease (SCD) and non transfusion dependent thalassaemia (NTDT) continue to evolve as our knowledge and clinical experience increases. Improved chelation options including drug combinations and a better understanding of condition specific factors may help to improve efficiency of chelation regimens and meet the needs of patients more effectively.
\end{abstract}

\section{Introduction}

There has been much progress in our understanding of iron overload and its consequences and the aims of chelation therapy are now focussed on the prevention of iron accumulation and irreversible organ damage. Recommendations for optimal use of chelation therapy in transfusion dependent thalassaemia, sickle cell disease and NTDT derive from many sources. The evidence base is continuing to evolve and consensus statements and guidelines have had a positive impact on patient outcomes.

\section{Iron balance and condition specific factors}

In different red cell conditions, the rate of iron accumulation and the distribution varies. In TDT untreated iron overload consequent to

Correspondence: Banu Kaya, Barts Health NHS Trust, Royal London Hospital, London, UK

E-mail: Banu.Kaya@bartsandthelondon.nhs.uk

Key words: iron toxicity; thalassaemia; sickle cell disease; chelation therapy; drug.

CC Copyright B. Kaya, 2014

Licensee PAGEPress, Italy

Thalassemia Reports 2014; 4:4860

doi:10.4081/thal.2014.4860

This article is distributed under the terms of the Creative Commons Attribution Noncommercial License (by-nc 3.0) which permits any noncommercial use, distribution, and reproduction in any medium, provided the original author(s) and source are credited. regular transfusions leads to liver damage, heart failure and endocrine dysfunction with a significant risk of death particularly from cardiac complications. The risk of iron loading in sickle cell disease is unlikely without regular transfusions but many patients require intermittent transfusions for management of complications. The rate of iron loading in SCD is also influenced by the transfusion regimen, and exchange transfusion regimens can significantly prevent risk of iron loading. The goal of chelation in SCD is therefore focussed on preventing liver iron loading. The risk of multisystem chronic organ dysfunction in SCD needs to be noted when prescribing chelators which may increase risk of damage, particularly renal and liver. With NTDT iron loading can occur despite minimal transfusion requirement. This has been linked in part to the significant ineffective erythropoesis and increased intestinal iron absorption.

\section{Licensed drugs and indications}

Three iron chelators, Deferoxamine (DF0), Deferiprone (DFP), and Deferasirox (DFX) are currently licensed for treating iron overload in TDT, though with variations in age of initiation or as first/second line therapy depending on the licensing authority. DFX in addition is registered for use in NTDT >age 10. Two of these, DFX and DFO are also licensed for SCD. The licensing at present applies to monotherapy though there is increasing evidence to support combination therapies in certain clinical contexts. For most prescribers, the licensing recommendations provide the initial guide for treatment.

\section{Practical aspects}

The optimal management of chelation therapy requires an understanding of the need to evaluate and revaluate the needs of the individual, changing situations and view this as a dynamic process. At initiation, an accurate evaluation of the transfusion history is a good starting point, with emphasis on avoiding delay in initiation, monitoring of compliance, side effects and complications and making optimal dose adjustments to treatment as required. During this continuation phase, careful monitoring of outcomes with serial serum ferritin (SF) measurements, liver iron concentration (LIC) quantitation and cardiac MR T2* assessments should be made and a decision made in conjunction with the patient or parents to continue or change to alternatives including combination therapies. In this time it may be necessary to intensify treatments, e.g., in preparation for pregnancy when a period of interruption will be necessary. Over time if the rate of iron input exceeds the output then rescue and emergency therapy may be required for excessive iron loading and acute organ decompensation. Contributing factors to inadequate chelation include side effects and compliance issues and these continue to be a concern. 


\section{Basic principles of regimens}

Licensed prescribing information relates to monotherapy with any of the three chelators but there is increasing experience in using combination therapies which can be simultaneous, sequential or alternate. In TDT, most experience applies to use of DFO and DFP together but use of combinations of the oral iron chelators is gaining interest with favourable results reported in the literature. There is little data on use of combination therapies in SCD.

\section{Evidence base and guidelines}

Recommendations for use of chelation therapy in TDT, SCD and NTDT continue to evolve as our understanding expands. This has been facilitated in practice by increasing high quality research exploring the efficacy, safety and tolerability of chelation regimens and monitoring methods in addition to experience gained from observational studies and expert opinion. This guidance has culminated in improving outcomes and quality of life. In practice, resource limitations and availability also impact on management and these factors also require consideration. In recent years a number of Cochrane reviews have explored use of DFO (Fischer et al., 2013), DFP (Fischer et al., 2013) and DFX (Meerpohl et al., 2012) in TDT and DFX (Meerpohl 2014) in SCD. A number of other meta-analyses have also been attempted (Kuo et al., Maggio et al., Mamtani et al., Xia et al.). These reviewed aggregated patient data but comparisons were difficult due to methodological differences between the studies. Cochrane systematic reviews analyse available data exclusively from randomised controlled trials (RCTs) therefore a large swath of published data is excluded. Trials in these conditions can be challenging because testing end points such as death, heart failure and cirrhosis require decades and also pose ethical issues. Patient preferences can also complicate randomisation. Current guidelines and consensus statements on the management of haemoglobinopathies all include recommendations for chelation therapy. Generally there is consistency but some variations exist, particularly around choice of chelator and in relation to combination therapy with DFO and DFP, (Musallam et al.). Guidelines with recommendations based on this growing evidence base require continual updating and many of the current guidelines are undergoing review.

\section{Thalassaemia}

DFO has been used extensively in this group of patients and the evidence from mainly retrospective cohort data implies a long term benefit with improved survival and reduced morbidity with regular use. The Cochrane collaboration reviewed use of DFO for managing transfusional iron overload in people with transfusion dependent thalassaemia. A total of 22 RCTs involving 2187 participants (range 11 to 586 people) were included up to March 2013. Overall, few trials measured the same outcomes and were not able to evaluate the risk of bias but 4 trials suggested an advantage of combining DFO with DFP to reduce iron stores as determined by SF. There was no advantage for LIC. In two trials a greater reduction in LVEF was noted with combination therapy compared to DFO monotherapy but there were differences in baseline LVEF between the groups.

The authors found all three available chelators produced significant reductions in iron loading but concluded DFO remain first line therapy for treating iron overload in TDT. They suggested the oral iron chelators are used as second line therapy. Intensification of DFO or consid- eration of combination therapy with DFO and DFP should be considered in patients with increasing myocardial iron. They did note however, note an increased rate of complications with combination therapy.

\section{Initiation and continuation in children}

DFO continues to be recommended for use as first line therapy in children but there is considerable evidence to promote first line therapy of DFX. A dose dependent effect on LIC, (Cappellini et al., 2011, Taher 2011) and myocardial T2*, (Pennell et al., 2012) have been noted. Two multicentre prospective studies assessing the safety and efficacy of DFP found a benefit in reducing SF at higher doses, (El-Alfy et al., 2010, Viprakasit et al., 2013). At present the data is limited to make recommendations for first line use of DFP but remains alternative therapy for children unable to manage with DFO or DFX. There is some evidence for the use of combination therapy with DFO and DFP in children and this can be considered as an option when first line therapy has failed. Few recommendations can be made for combination therapies using DFX and DFO or DFX and DFP but preliminary data are encouraging.

\section{Continuation in adults}

Continual assessment of transfusion requirement, body iron burden and in particular evidence of cardiac iron loading is necessary in adult patients, (Brittenham 2011). For those patients who are able to maintain neutral iron balance and who have an acceptable quality of life with one of the three iron chelators, no deviation from preferred monotherapy is required. There is longstanding survival data for DFO, (Borgna-Pignatti 2004) and 5 year trial data for DFX demonstrates efficacy in reducing iron burden with an acceptable side effect profile, (Cappellini 2011). Data from prospective trials suggest a preservation of LVEF, and low risk of progression to heart failure with DFX or DFO, (Pennell et al., 2014) in high risk individuals who have preserved LVEF. However for individuals who have evidence of severe myocardial iron and heart failure, the American Heart Association (Pennell et al., 2013) recently issued guidance for the management of patients and suggested intensified continuous DFO administration with early introduction of DFP. Indications for change are summarised in the recent Thalassaemia International Federation (TIF) guidelines: where there is evidence of increasing or high iron loading, consideration should be given to dose and frequency increase, other monotherapies or combination regimens. Any increase in cardiac iron loading and cardiac status should be taken into consideration when making changes and attention given to adjustments for low body iron. Alternative combination therapies are also being explored though there are no clear recommendations at present, (Cassinerio et al., 2014).

\section{Sickle cell disease}

The aims of chelation therapy in SCD are similar to thalassaemia and guidelines for SCD have often been extrapolated from those used for thalassaemia, with similar recommendations for initiation; namely consideration given to transfusion usage, serum ferritin and liver iron concentration (LIC). The limitations of serum ferritin measurements in SCD are recognised. Due to the lesser likelihood of extrahepatic iron loading, the emphasis has been on preventing liver complications with less clear guidance on extrahepatic complications including cardiac monitoring. The evidence base to support chelation options in SCD is also increasing 
with at least 10 trials with more than 4 participants evaluating the three available iron chelators. The safety and effectiveness of iron chelation in SCD was reported in a large prospective clinical trial of DFX where patients completing a 1 year, phase II, randomised, DFO controlled study, entered a 4 year extension, continuing to receive DFX or switching from DFO to DFX (Vichinsky et al., 2011). A Cochrane review (Meerpohl et al., 2014) concluded that DFX had a similar efficacy to DFO and an acceptable short term safety profile. There is least experience with using DFP. Results from a recently published 5-year multicentre randomised trial comparing DFP with DFO found both chelators effective at reducing serum ferritin with an acceptable safety profile (Calvaruso et al.,). The specific characteristics of SCD, including risk of renal disease make the use of chelation therapy particularly challenging and further trials are required to guide further recommendations.

\section{Non transfusion dependent thalassaemia}

Generally recommendations for NTDT derive from experience in TDT. TIF guidelines for the management of NTDT suggest initiation of chelation therapy in patients who are older than 10 years and have LIC $>5 \mathrm{mg} / \mathrm{g}$ dry weight. They suggest initiation with DFX at low dose with appropriate monitoring. Recently SF measurements have been suggested to guide therapy (Taher et al., 2014).

\section{Special situations}

There remain some uncertainties around the management of particular clinical scenarios. These include chronic kidney disease in SCD where both DFO and DFX have been used in experienced centres but data on use is limited. Currently the oral iron chelators are contraindicated in pregnancy and DFO only advised in the second and third trimesters. Few studies explored use of the oral iron in very young children (< age 2) and the current licensing does not recommend using in this age group. With good compliance many patients attain low iron burden. There are recommendations in place for careful monitoring and dose adjustment and some evidence to promote intensive chelation in adults but few prospective trials particularly in children to routinely recommend this.

\section{Conclusions}

There is increasing evidence supporting chelation therapy as an effective treatment for transfusional iron overload to improve survival and reduce the risk of complications including heart failure. Inadequate chelation remains a concern however due to side effects and compliance. Recommendations on initiation, continuation or intensification are continuing to evolve as more data on individual and combination therapies becomes available. Combination therapies with oral iron chelators may be more preferable for patients and help with compliance. Uncertainties though remain around optimal management particularly in special circumstances.

\section{References}

Borgna-Pignatti C, Rugolotto S, De Stefano P, et al. Survival and complications in patients with thalassemia major treated with transfusion and deferoxamine. Haematologica 2004;89:1187-93.
Brittenham GM. Iron-chelating therapy for transfusional iron overload. N Engl J Med 2011;364:146-56.

Calvaruso G, Vitrano A, Di Maggio R, et al. Deferiprone versus deferoxamine in sickle cell disease: results from a 5-year long-term italian multi-center randomized clinical trial. Blood Cells Mol Dis 2014;53:265-71.

Cappellini MD, Bejaoui M, Agaoglu L, et al. Iron chelation with deferasirox in adult and pediatric patients with thalassemia major: efficacy and safety during 5 years' follow-up. Blood 2011;118:884-93.

Cassinerio E, Orofino N, Roghi A, et al. Combination of deferasirox and deferoxamine in clinical practice: an alternative scheme of chelation in thalassemia major patients. Blood Cells Mol Dis 2014;53:164-7.

Elalfy MS, Sari TT, Lee CL, et al. The safety, tolerability, and efficacy of a liquid formulation of deferiprone in young children with transfusional iron overload. J Pediatr Hematol Oncol 2010;32:601-5.

Fisher SA, Brunskill SJ, Doree C, et al. Desferrioxamine mesylate for managing transfusional iron overload in people with transfusiondependent thalassaemia. Cochrane Database Syst Rev 2013;21;8:CD004450.

Fisher SA, Brunskill SJ, Doree C, et al. Oral deferiprone for iron chelation in people with thalassaemia. Cochrane Database Syst Rev 2013;21;8:CD004839.

Kuo KH, Mrkobrada M. A systematic review and meta-analysis of deferiprone monotherapy and in combination with deferoxamine for reduction of iron overload in chronically transfused patients with $\beta$-Thalassemia. Hemoglobin 2014;13:1-13.

Maggio A, Filosa A, Vitrano A, et al. Iron chelation therapy in thalassemia major: a systematic review with meta-analyses of 1520 patients included on randomized clinical trials. Blood Cells Mol Dis 2011;47:166-75.

Mamtani M, Kulkarni H. Influence of iron chelators on myocardial iron and cardiac function in transfusion-dependent thalassaemia: a systematic review and meta-analysis. Br J Haematol 2008 Jun;141:882-90.

Meerpohl JJ, Antes G, Rücker G, et al. Deferasirox for managing iron overload in people with thalassaemia. Cochrane Database Syst Rev 2012;2:CD007476.

Meerpohl JJ, Schell LK, Rücker G, et al. Deferasirox for managing transfusional iron overload in people with sickle cell disease. Cochrane Database Syst Rev 2014;5:CD007477.

Musallam KM, Angastiniotis M, Eleftheriou A, Porter JB. Cross-talk between available guidelines for the management of patients with $\beta$-thalassemia major. Acta Haematol 2013;130:64-73.

Pennell DJ, Porter JB, Cappellini MD, et al. Deferasirox for up to 3 years leads to continued improvement of myocardial $\mathrm{T} 2 *$ in patients with $\beta$-thalassemia major. Haematologica. 2012;97:842-8.

Pennell DJ, Porter JB, Piga A, et al. A 1-year randomized controlled trial of deferasirox vs deferoxamine for myocardial iron removal in $\beta$ thalassemia major (CORDELIA). Blood 2014;123:1447-54.

Pennell DJ, Porter JB, Piga A, et al.; on behalf of the CORDELIA study investigators. Sustained improvements in myocardial T2* over 2 years in severely iron-overloaded patients with $\beta$ thalassemia major treated with deferasirox or deferoxamine. Am J Hematol 2014.

Pennell DJ, Udelson JE, Arai AE, et al. Cardiovascular function and treatment in $\beta$-thalassemia major: a consensus statement from the American Heart Association. Circulation 2013;128:281-308.

Taher A, Elalfy MS, Al Zir K, et al. Importance of optimal dosing $\geq 30$ $\mathrm{mg} / \mathrm{kg} / \mathrm{d}$ during deferasirox treatment: 2.7-yr follow-up from the ESCALATOR study in patients with $\beta$-thalassaemia. Eur J Haematol 2011;87:355-65.

Taher AT, Porter JB, Viprakasit V, et al. Defining serum ferritin thresholds to predict clinically relevant liver iron concentrations for guiding deferasirox therapy when MRI is unavailable in patients with 
non-transfusion-dependent thalassaemia. Br J Haematol 2014.

Vichinsky E, Bernaudin F, Forni GL, et al. Long-term safety and efficacy of deferasirox (Exjade) for up to 5 years in transfusional iron-overloaded patients with sickle cell disease. $\mathrm{Br} \mathrm{J}$ Haematol 2011;154:387-97.

Viprakasit V, Nuchprayoon I, Chuansumrit A, et al. Deferiprone (GPO$\mathrm{L}-\mathrm{ONE}(\circledR)$ monotherapy reduces iron overload in transfusiondependent thalassemias: 1-year results from a multicentre prospective, single arm, open label, dose escalating phase III pediatric study (GPO-L-ONE; A001) from Thailand. Am J Hematol 2013;88:251-60.

Xia S, Zhang W, Huang L, Jiang H. Comparative efficacy and safety of deferoxamine, deferiprone and deferasirox on severe thalassemia: a meta-analysis of 16 randomized controlled trials. PLoS One 2013;8:e82662. 